\title{
Can psychobiotics intake modulate psychological profile and body composition of women affected by normal weight obese syndrome and obesity? A double blind randomized clinical trial
}

Antonino De Lorenzo ${ }^{1,8^{*}}$, Micaela Costacurta², Giuseppe Merra ${ }^{3}$, Paola Gualtieri ${ }^{4}$, Giorgia Cioccoloni ${ }^{4}$, Massimiliano Marchetti ${ }^{5,6}$, Dimitrios Varvaras ${ }^{7}$, Raffaella Docimo ${ }^{2}$ and Laura Di Renzo ${ }^{1}$

\begin{abstract}
Background: Evidence of probiotics effects on gut function, brain activity and emotional behaviour were provided. Probiotics can have dramatic effects on behaviour through the microbiome-gut-brain axis, through vagus nerve. We investigated whether chronic probiotic intake could modulate psychological state, eating behaviour and body composition of normal weight obese (NWO) and preobese-obese (PreOB/OB) compared to normal weight lean women (NWL).

Methods: 60 women were enrolled. At baseline and after a 3-week probiotic oral suspension (POS) intake, all subjects underwent evaluation of body composition by anthropometry and dual X-ray absorptiometry, and psychological profile assessment by self-report questionnaires (i.e. EDI-2, SCL9OR and BUT). Statistical analysis was carried out using paired t test or a non-parametric Wilcoxon test to evaluate differences between baseline and after POS intake, one-way ANOVA to compare all three groups and, where applicable, Chi square or t test were used to assess symptoms.
\end{abstract}

Results: Of the 48 women that concluded the study, 24\% were NWO, 26\% were NWL and 50\% were PreOB/OB. Significant differences in body composition were highlighted among groups both at baseline and after a POS ( $p<0.05$ ). After POS intake, a significant reduction of BMI, resistance, FM ( $\mathrm{kg}$ and \%) $(\mathrm{p}<0.05)$, and a significant increase of FFM ( $\mathrm{kg}$ and \%) $(\mathrm{p}<0.05)$ were observed in all subjects in NOW and PreOB/OB. After POS intake, reduction of bacterial overgrowth syndrome $(p<0.05)$ and lower psychopathological scores $(p<0.05)$ were observed in NWO and PreOB/ OB women. At baseline and after POS intake, all subjects tested were negative to SCL90R_GSI scale, but after treatment subjects positive to BUT_GSI scale were significantly reduced $(8.33 \%)(p<0.05)$ compared to the baseline (33.30\%). In NWO and PreOB/OB groups significant differences $(p<0.05)$ in response to the subscales of the EDI-2 were observed. Significant improvement of the orocecal transit time was observed $(p<0.05)$ after POS intake. Furthermore, significant differences were observed for meteorism $(p<0.05)$ and defecation frequency $(p<0.05)$.

Conclusions: A 3-week intake of selected psychobiotics modulated body composition, bacterial contamination, psychopathological scores of NWO and PreOB/OB women. Further research is needed on a larger population and for a longer period of treatment before definitive conclusions can be made.

\footnotetext{
*Correspondence: delorenzo@uniroma2.it

${ }^{8}$ Section of Clinical Nutrition and Nutrigenomic, Department

of Biomedicine and Prevention, University of Rome Tor Vergata, Via

Montpellier 1, 00136 Rome, Italy

Full list of author information is available at the end of the article
} 
Trial registration ClinicalTrials.gov Id: NCT01890070

Keywords: NWO syndrome, Probiotic, Psychobiotic, Psychological profile, Body composition

\section{Background}

The human gut hosts a dynamic and complex microbial ecosystem. It is represented by approximately $1 \mathrm{~kg}$ of bacteria in the average adult, about the weight of the human brain. It is composed by microorganisms belonging to 14 families, 45 genera and 400-500 distinct species, variously distributed along the entire intestinal tract. Two bacterial phylotypes Firmicutes and Bacteroidetes are the most abundant bacteria species in the gut [1].

The microbiota-gut brain axis, recently termed "psychobiota" [2], plays an important role in host physiology, as in the regulation of neuroinflammation, neuroendocrine stress response, neurodevelopment and modulation of mood and behavior [3-5], which implicates it in psychiatric disorders, such as stress, anxiety and depression.

Nowadays, several studies identify the relationship between gut microbiota and the onset of various diseases, demonstrating the ability of probiotics in controlling inflammatory processes, attenuation of metabolic dysfunctions, normalization of stress-induced abnormal behaviors, regulation of the hypothalamus-pituitaryadrenal axis (HPA axis) and neuropsychiatric disorders [6-8].

The evidence of the inflammatory state alteration, highlighted in schizophrenia, major depressive disorder and bipolar disorder, strongly recalls the microbiota alteration, suggesting an important role of the alteration of the gastrointestinal (GI) system also in neuropsychiatric disorders [8].

Probiotics can have dramatic effects on behaviour, through their action on the vagus nerve and on the microbiome-gut-brain axis, which constitutes a bidirectional communication network. Probiotics produce a variety of neurochemicals, analogues of mammalian hormones, involved in mood and behaviour. Therefore, the visceral messages from the gut can affect brain function and, vice versa, signals from the brain may affect the sensory system and the gut secretion mode.

The bacteria most commonly exploited as probiotics with psychotropic effects in animal models and human clinical trials have been classified as "psychobiotics" [2], and they belong to the Bifidobacterium and Lactobacillus families.

In the last few years, in vivo experiments demonstrated that administration of Bifidobacterium longum 1714 and Bifidobacterium breve 1205, Lactobacillus helveticus and Lactobacillus plantarum strain PS128 reduce anxiety and depression like behaviors, HPA axis hyperactivity and abnormal neurochemical changes [9].

Gut microbiota seems to have an impact on the serotonergic system. In fact, treatment with Lactobacillus helveticus in hyperammonemia-treated rats led to an anxiolytic effect and improved cognitive function possibly through a reduction in hippocampal 5-Hydroxy Tryptamine (5HT) levels [10].

Moreover, a combined treatment with Lactobacillus helveticus and rhamnosus prevented intestinal permeability alteration caused by stress [11].

Anxiety-like behavior associated with chronic colitis in mice was attenuated by $B$. longum administration, associated with decreased hippocampal brain-derived neurotrophic factor (BDNF) mRNA $[12,13]$.

Although less evidence exists for probiotic actions on human population, it has been demonstrated that patients who received a probiotic formulation containing Lactobacillus acidophilus, Lactobacillus casei and Bifidobacterium bifidum, significantly decreased Beck Depression Inventory (BDI) scores [14]. Moreover, a probiotic mixture of Lactobacillus helveticus and Bifidobacterium longum and a probiotic-containing milk drink of Lactobacillus casei showed positive effects on psychological distress $[15,16]$.

Furthermore, a growing body of scientific evidence supports the notion that the crosstalk between the gut microbiota, diet and immune system activates mediators and signalling pathways, which influence the whole body metabolism and disease [17-19]. The metabolic activities of the gut microbiota play a decisive part in obesity because of its role in the improvement of calorie extraction from food, the accumulation of substrates in adipose tissue, like fatty acids, and the utilization of energy and nutrients for its growth [20].

Obesity is characterized by a peculiar microbiota and the microbiota itself, together with the host genotype and lifestyle, could contribute to the development of this metabolic dysfunction. Also, a bidirectional association between obesity and self-reported or clinical measures of depression were observed, and body dissatisfaction was robustly associated as a risk factor for obesity and eating disorders [9].

Different obese phenotypes have been described based on body fat composition and distribution, rather than the simple increase of body weight, body mass index (BMI) and genetics [21]: (1) metabolically obese normal weight [22]; (2) metabolically healthy obese [23]; and (3) 
metabolically unhealthy obese or "at risk" obese [24]; (4) normal weight obese (NWO) [25].

Subjects affected by NWO syndrome usually have a BMI value within the normal range $\left(<25 \mathrm{~kg} / \mathrm{m}^{2}\right)$ and, at the same time, high total body fat percentage (TBFat $>30 \%$ ) and total body lean mass (TBLean) deficiency based on a genetic predisposition. They also have high oxidative stress level, early inflammatory status and some metabolic abnormalities [26-28]. Furthermore, in our previous study on female subjects affected by NWO syndrome it was highlighted, not only an increased risk of cardiovascular and metabolic disease, but also a will to control body weight, revealing a suppressed vocation for obesity. In fact, NWO subjects obtained an intermediate score on the eating disorder inventory-2 (EDI-2), particularly in terms of body dissatisfaction and drive for thinness, between normal weight lean women and pre-obese or obese women [28].

Up today very few studies demonstrated the beneficial effects on the health status of obese subjects with psychiatric illnesses and eating disorders of these "mind-altering" probiotics. Lactobacillus appears to reduce body fat mass, anxiety and dysphoria, and improves insulin sensitivity and glucose tolerance $[29,30]$.

Nowadays, literature doesn't report any paper focused on the effectiveness check of treatment with psychobiotics in NWO syndrome.

Given the link among gut microbiota, body composition, the risk of eating disorder, anxiety- and depressionlike behaviours, the purpose of the current study was to test the efficacy of a 3-week administration of a psychobiotic oral suspension (POS), containing Streptococcus thermophilus, Bifidobacterium animalis subsp. Lactis, Streptococcus thermophiles, Bifidobacterium bifidum, Lactobacillus delbrueckii spp. Bulgaricus, Lactococcus lactis subsp. Lactis, Lactobacillus acidophilus, Lactobacillus plantarum, Lactobacillus reuteri, selected according to literature. The endpoints of this study were to evaluate the body composition parameters, the psychological profile, and eating behaviour changes of NWO and preobese-obese (PreOB/OB) compared to normal weight lean women (NWL), after POS.

\section{Methods}

\section{Clinical study design and subjects}

The clinical study was conducted using a randomized, double blinded controlled design, between October 2015 and July 2016, according to the CONSORT flowchart (Fig. 1). Subjects were consecutively recruited within a program of routine medical check-up at the Section of Clinical Nutrition and Nutrigenomic, Department of Biomedicine and Prevention of the University of Rome "Tor Vergata", at "Nuova Annunziatella" Clinic, and General Hospital Foundation, Catholic University of Sacred Heart, Rome, Italy.

Sixty women were enrolled. Subjects were screened for eligibility at first medical visit and underwent body composition analysis, psychological profile and eating behaviour assessment by symptom checklist 90 (SCL90R), [31] for the evaluation of general psychopathology, body uneasiness test (BUT), for the evaluation of body image perception (IC) [32], and EDI-2, for eating behavior [33], in order to test the efficacy of a 3-week administration of POS on NWO and preobese-obese (PreOB/OB) compared to normal weight lean women (NWL).

As the expected weight loss was below 5 , according the Ethical Committee of the University of Rome "Tor Vergata", data from Dual X-ray Absorptiometry (DXA) was used to classified the subjects. We classified the subjects according to BMI, and TBFat \% by DXA into: (a) NWL women, with a BMI $<25 \mathrm{~kg} / \mathrm{m}^{2}$ and TBFat $\%<30$; (b) NWO women, with a BMI $<25 \mathrm{~kg} / \mathrm{m}^{2}$, and TBFat $\% \geq 30$; (c) PreOB/OB women, with a BMI $\geq 25 \mathrm{~kg} / \mathrm{m}^{2}$ and TBFat $\% \geq 30$.

Body composition was assessed by Bioelectrical Impedance Analysis (BIA) at baseline and after treatment.

Forty-eight subjects were eligible for the study and they were randomly divided into two groups (1:1 ratio). The randomization was determined by an external contract research organization and coordinated with the Section of Clinical Nutrition and Nutrigenomic, at the University of Rome "Tor Vergata", independently of the investigators.

One intervention group (IG) and one control group (CG) were utilized and took daily n.1 bag of POS, or n.1 bag of placebo. The study consists of a 3-week treatment, with POS or placebo, separated by a 3-week wash out period, used to avoid additive effects on treatments to follow. After 3 weeks of washout, the IG and CG were reversed. The IG and CG arms were double-blinded. Study period resulted in a total duration of 9 weeks. At the beginning and at the end of each arm ( \pm 3 days), the subjects had to repeat the visit.

Subjects were asked to maintain their usual lifestyle habits and to report any illness or abnormality arising during the study. At the end of each arm, a clinician assessed any adverse effects from the interventions by going through a checklist of symptoms, including bloating, fullness or indigestion, altered bowel habit, dizziness and other symptoms that were possibly associated with the interventions. Any adverse effect has been properly reported.

\section{Exclusion criteria}

Exclusion criteria included age $<20$ or $>65$ year, pregnancy, breast-feeding, type 1 diabetes, presence of intestinal bacterial overgrowth, characterized by high levels 


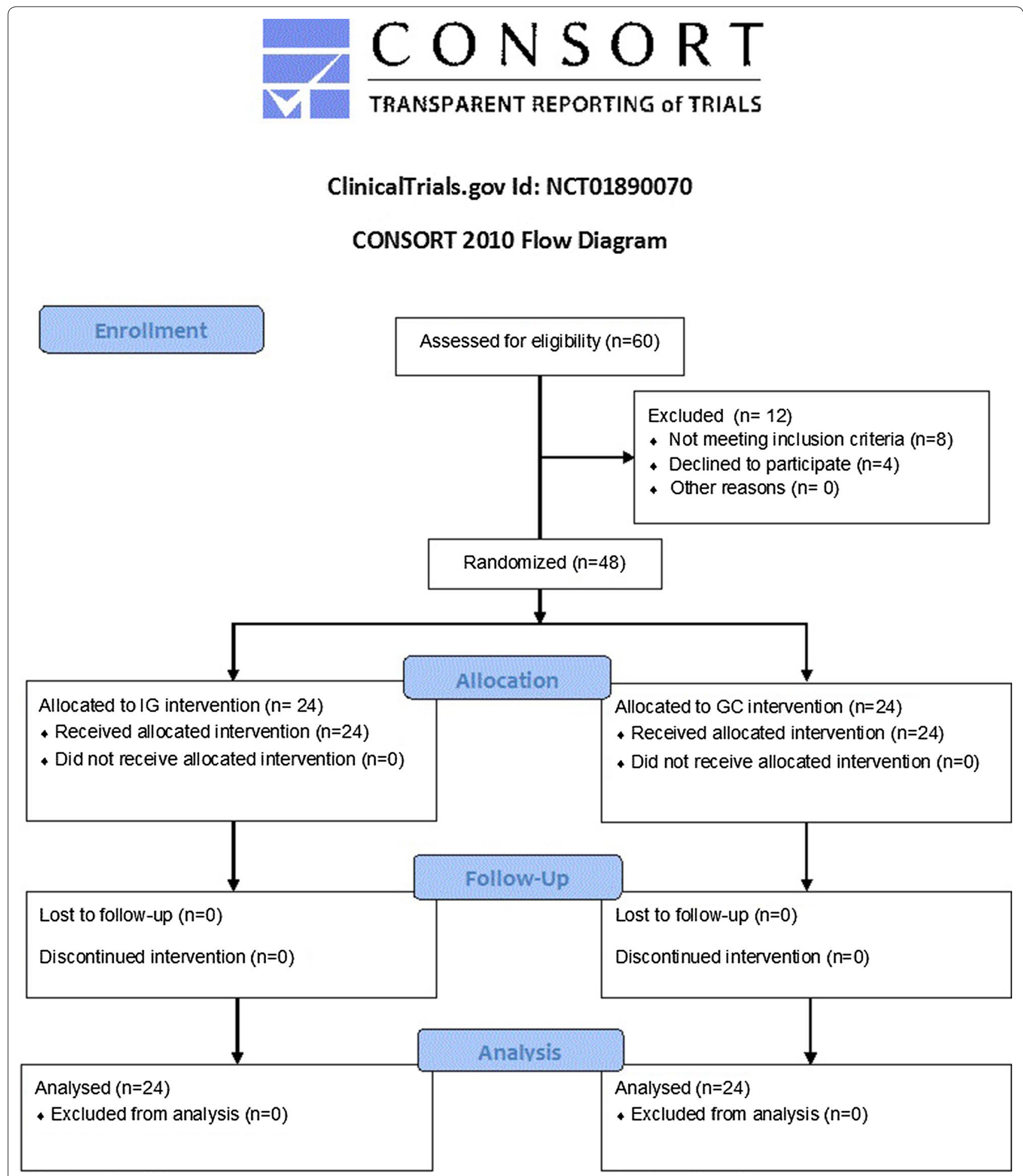

Fig. 1 Study Flow Diagram according to Consort, 2010

of hydrogen and methane production in the small bowel, acute diseases, endocrine disorders, liver, heart or kidney dysfunctions, history of chronic degenerative or infectious diseases, medication, antibiotic therapy until
10 days before enrolment, smoke, drug or alcohol abuse, participation in another diet trial. No subjects with known alterations to intestinal transit following organic pathologies (abdominal surgery, diabetes mellitus, 
scleroderma, hypothyroidism, etc.) were included in the study. Subjects could not have taken antibiotics or probiotics in the month before the study and were willing to avoid use of probiotics for the duration of the study.

\section{Endpoints}

The primary endpoint was the evaluation of body composition changes after POS by anthropometry, DXA, and bioimpedentiometry. The secondary endpoint was the evaluation of psychological profile by self-report questionnaires (i.e. EDI-2, SCL90R, and BUT). The third endpoint was the evaluation of orocaecal transit time and bacterial overgrowth by lactulose breath test (LBT).

\section{Psychobiotics oral suspension (POS) and placebo capsules composition}

1 bag of 3 g psychobiotics oral suspension (POS) contained: Streptococcus thermophilus SGSt01 $\left(1.5 \times 10^{10}\right.$ colony-forming unit CFU), Bifidobacterium animalis subsp. Lactis SGB06 $\left(1.5 \times 10^{10}\right.$ colony-forming unit CFU), Streptococcus thermophiles $\left(1.5 \times 10^{10}\right.$ colonyforming unit CFU), Bifidobacterium bifidum SGB02 $\left(1.5 \times 10^{10}\right.$ colony-forming unit CFU), Lactobacillus delbrueckii spp. Bulgaricus DSM $20081\left(1.5 \times 10^{10}\right.$ colony-forming unit CFU), Lactococcus lactis subsp. Lactis SGLC01 $\left(1.5 \times 10^{10}\right.$ colony-forming unit CFU), Lactobacillus acidophilus SGL11 $\left(1.5 \times 10^{10}\right.$ colony-forming unit CFU), Lactobacillus plantarum SGLO7 $\left(1.5 \times 10^{10}\right.$ colony-forming unit CFU), Lactobacillus reuteri SGL01 $\left(1.5 \times 10^{10}\right.$ colony-forming unit CFU), maltodextrin from corn, anti-caking agent (silica), casein, lactose and gluten $<3$ ppm LLOQ (lower limit of quantitation) (Biocult strong, HOMEOSYN, Rome, Italy).

The placebo was represented by $3 \mathrm{~g}$ of inert material (flour type 00), maltodextrin from corn, anti-caking agent (silica). The appearance of the placebo was indistinguishable in colour, shape, size, packaging, smell, and taste from that of the probiotic supplement.

Both products were provided by HOMEOSYN (Rome, Italy).

\section{Anthropometric measurements}

At T1, after a 12-h overnight fast, all subjects underwent anthropometric evaluation (body weight, height, waist and hip circumferences), according to standard method [34]. All the individuals were instructed to take off their clothes and shoes before undergoing the measurements.

BMI was calculated using the formula: $\mathrm{BMI}=$ body weight $(\mathrm{kg}) /$ height $(\mathrm{m})^{2}$.

\section{Bioelectrical impedance analysis (BIA)}

Resistance, reactance, impedance, phase angle, total body water (TBW), intracellular water (ICW), extracellular water (ECW), free fat mass (FFM) and fat mass (FM) were measured using a BIA phase sensitive system at $50 \mathrm{kHz}$ frequency (BIA 101S, Akern/RJL Systems-Florence, Italy).

Measurements were taken according to Di Renzo et al. [35].

\section{Dual x-ray absorptiometry}

To assess body composition analysis, that give the possibility to measure TBFat and TBLean, DXA (i-DXA, GE Medical Systems, Milwaukee, WI, USA) evaluation was performed at baseline, according to De Lorenzo et al. [28].

\section{Psychodiagnostic instruments}

Anonymous questionnaires, self-compiled, were given to all subjects for the collection of socio-demographic data. The symptom checklist 90 (SCL90R) [31] was administered for the evaluation of general psychopathology, the body uneasiness test (BUT) for the evaluation of the perception of body image (IC) [32] and the EDI-2, for eating behavior [33]. Eating behavior was assessed using the Italian version of the EDI-2, standardized in an Italian population [36]. The subscales of the EDI-2 include drive for thinness (DT), bulimia (B), body dissatisfaction (BD), ineffectiveness (I), interceptive awareness (IA), maturity fears (MF), asceticism (A), impulse regulation (IR), social insecurity (SI), perfectionism (P) and interpersonal distrust (ID).

Body uneasiness test (BUT) It is a self-assessment scale, used for body image studies and related pathologies. Beyond the total score, BUT allows to calculate the global severity index (GSI) or total average score, which is obtained from the sum of clinical scores (BUT a), divided by their number (34). Items number with score $\geq 1$ correspond to positive symptom total (PST). The sum of items scores $\geq 1$ divided by PST, produces the positive symptom distress index (PSDI) [32].

Five factors were defined: WP-weight phobia, BICbody image concerns, A-avoidance, CSM-compulsive self-monitoring, D-depersonalization. In our study, we considered as positive for altered perception of body image a GSI score $\geq 1.2$.

Symptom check list-revised (SCL90R) It is a general evaluation scale of the psychopathology, based on patient's self-evaluation. This scale is composed by 90 items, which investigate the presence of symptoms in the week before the test check. These 90 items, which have five levels Likert answers, have 10 reference factors: (1) somatization (Som); (2) obsessive/compulsive (Obs); (3) interpersonal sensitivity (Interp Sens); (4) depression (Dep); (5) anxious 
(Anx); (6) anger/hostility (Anger Host); (7) phobia (Phob); (8) psychoticism (Psych); (9) paranoia (Paran); (10) sleep disorders. The score goes from 0 to 4 , and a score above 1 is an index of pathology [31].

\section{Breath testing and gastrointestinal symptoms questionnaire}

Subjects also completed a questionnaire evaluating gastrointestinal symptoms (meteorism, abdominal pain, defecation frequency/week).

The LBT was performed by administering $20 \mathrm{~g}$ lactulose dissolved in $100 \mathrm{cc}$ water to the subjects. Breath samples were obtained by asking the patients to blow into suitable containers at time 0 (before ingesting the lactulose) and then every $15 \mathrm{~min}$ thereafter for the $4 \mathrm{~h}$ following lactulose administration. Gas chromatography was used to assess the presence and quantity of hydrogen in the breath (Quintron Milwaukee, Wisconsin USA). Orocaecal transit time was calculated for each patient by constructing the curves of hydrogen in the breath over time. This therefore showed the time necessary for the bolus to reach the caecum [37].

\section{Statistical analysis}

The statistical analysis was carried out using IBM SPSS Statistics for Windows, Version 21.0 (Armonk, NY: IBM Corp). Data are expressed as mean \pm standard deviation (SD), and minimum and maximum. A paired test or a non-parametric Wilcoxon test were performed to evaluate differences between baseline and after POS. A one-way ANOVA was carried out to compare the average of the responses obtained in all three groups. Where applicable, the Chi square or Student's t test were used to assess symptoms.

A difference of $\mathrm{p}<0.05$ was considered significant.

\section{Results}

Of the 60 women initially recruited, 8 did not meet inclusion criteria, 4 dropped out of the study voluntarily, leaving a total of 48 subjects for final analysis.

The characteristics of the study population in terms of age, weight, height, BMI, body composition and psychological profile (EDI-2, BUT, and SCL90R) are shown in Table 1. In particular, between the NWL and the NWO groups, significant differences $(\mathrm{p}<0.05)$, in terms of weight $(\Delta \% 14.31)$, BMI $(\Delta \% 16.95)$, hip circumference $(\Delta \% 9.4)$, TBW (\%) $(\Delta \%-10.05)$, TBFat $(\%)(\Delta \%$ 32.77), TBFat (g) $(\Delta \% 43.43)$, FM $(\mathrm{kg})(\Delta \% 58.59)$, FM (\%) $(\Delta \% 39.75)$ and FFM $(\%)(\Delta \%-10.13)$ were observed. Between the NWL and the PreOB/OB there were significant differences $(\mathrm{p}<0.05)$ in terms of weight $(\Delta \% 25.06)$, BMI $(\Delta \% 35.22)$, waist $(\Delta \% 18.93)$ and hip circumference $(\Delta \% 14.50)$, PA $\left(^{\circ}\right)(\Delta \% 17.46), \mathrm{TBW}(\%),(\Delta \%-17.49)$,
ECW (\%) ( $\Delta \%-9.57), \mathrm{ICW}(\mathrm{L})(\Delta \% 11.61), \mathrm{ICW}(\%)(\Delta \%$ 7.65), TBFat (\%) ( $\Delta \%$ 64.97), TBFat (g) ( $\Delta \% 125.08)$, FM $(\mathrm{kg})(\Delta \% 64.32)$, FM $(\%)(\Delta \% 43.35)$ and FFM $(\%)(\Delta \%$ $-11.04)$. Significant differences $(\mathrm{p}<0.05)$ between the NWO and the PreOB/OB groups in terms of weight $(\Delta \%$ 9.40), BMI ( $\Delta \%$ 15.62), Reactance (Ohm), ( $\Delta \% 19.73)$, PA $\left(^{\circ}\right)(\Delta \% 20.72)$, TBW (L) $(\Delta \%-0.60)$, TBW $(\%)(\Delta \%$ $-8.27)$, ECW (L) $(\Delta \%-12.45)$, ECW $(\Delta \%-11.70)$, and ICW (L) ( $\Delta \%$ 9.50), ICW (\%) ( $\Delta \%$ 9.78), TBFat (\%) $(\Delta \%$ 24.26) and TBFat (g) ( $\Delta \%$ 56.93) were observed (Table 1$)$.

After 3 weeks of POS treatment, a significant reduction of BMI, resistance, reactance, PA, ICW (\%), FM (kg and \%) $(\mathrm{p}<0.05)$, and a significant increase of TBW (L and \%), ECW (L and \%), ICW (L), FFM (kg) and FFM (\%) were observed in NOW population. Furthermore, in PreobOB group it was highlighted a significant reduction of weight, BMI, waist, hip, resistance, ECW (L and \%), FM ( $\mathrm{kg}$ and \%), and a significant increase of PA, TBW (\%), ICW (L and \%), FFM ( $\mathrm{kg}$ and \%) $(\mathrm{p}<0.05)($ Table 1$)$.

Significant differences $(\mathrm{p}<0.05)$ were observed among the NWL, NWO and PreOB/OB groups in body composition parameters after POS treatment (Table 1).

At baseline, the total tested sample was negative to SCL90R_GSI scale and $33.30 \%$ of the population was positive at BUT_GSI scale (GSI $\geq 1.2$; mean $0.96 \pm$ standard deviation 0.66). After 3 weeks of POS treatment, all population remained negative to SCL90R_GSI scale, and the positive to BUT scale was significantly reduced $(\mathrm{p}<0.05)$ at $8.33 \%$ (GSI $\geq 1.2$; mean $0.59 \pm$ standard deviation 0.52 ). At baseline, among the $33.3 \%$ of the positive to BUT_GSI and BUT_CSM scale, the $12.5 \%$ were NWL, the $50 \%$ were NWO, and $37.55 \%$ were PreOB/OB. After POS treatment only NWO among the $8.33 \%$ of the positive (100\%) were identified.

The average scores of the various dimensions and the total score of SCL90R scale, BUT_GSI and EDI-2 are represented in Table 2. After 3 weeks of POS treatment, in the general population significant differences $(\mathrm{p}<0.05)$ in terms of the responses to the subscales of the EDI-2 were observed: $-37.98 \Delta \%$ of B (baseline, $1.74 \pm 3.01$; 3 weeks POS, $1.08 \pm 2.06$ ), the $-15.95 \Delta \%$ of DT (baseline, $1.74 \pm 3.01$; 3 weeks POS, $1.08 \pm 2.06$ ), the -40.15 $\Delta \%$ of I (baseline, $1.74 \pm 3.01$; 3 weeks POS, $1.08 \pm 2.06$ ). After POS treatment, in the NWO group significant differences $(p<0.05)$ in terms of the responses to the subscales of the EDI- 2 were observed: $-41.94 \Delta \%$ of $B$ (baseline, $0.91 \pm 1.64$; 3 weeks POS, $0.53 \pm 1.16$ ), the $-19.30 \Delta \%$ of DT (baseline, $9.29 \pm 7.81$; 3 weeks POS, $7.50 \pm 7.18$ ), the $-50.45 \Delta \%$ of I (baseline, $3.26 \pm 4.29$; 3 weeks POS, $1.62 \pm 2.85)$. After 3 weeks of POS treatment, in the PreOB/OB group significant differences, $(\mathrm{p}<0.05)$ in terms of responses to the subscales of the EDI-2, were observed: $-31.25 \Delta \%$ of B (baseline, 
Table 1 Comparison of body composition parameters of normal weight lean, normal weight obese and pre-obese/obese groups between baseline and after 3 weeks POS treatment

\begin{tabular}{|c|c|c|c|c|c|c|}
\hline & \multicolumn{2}{|l|}{ NWL } & \multicolumn{2}{|l|}{ NWO } & \multicolumn{2}{|l|}{ PreobOB } \\
\hline & Baseline & POS & Baseline & POS & Baseline & POS \\
\hline Age (years) & \multicolumn{2}{|c|}{$30.18 \pm 2.04(28.00-33.00)$} & \multicolumn{2}{|c|}{$40.00 \pm 12.56(27.00-56.00)$} & \multicolumn{2}{|c|}{$33.57 \pm 10.57(24.00-50.00)$} \\
\hline Height (cm) & \multicolumn{2}{|c|}{$164.64 \pm 4.03(160.00-170.00)$} & \multicolumn{2}{|c|}{$162.90 \pm 6.60(154.00-168.00)$} & \multicolumn{2}{|c|}{$158.33 \pm 1.86(155.60-160.00)$} \\
\hline Weight (kg) & $\begin{array}{r}55.55 \pm 4.65^{\mathrm{a}, \mathrm{o}} \\
(50.50-62.50)\end{array}$ & $\begin{array}{r}54.84 \pm 5.63^{X, \omega} \\
(49.20-63.00)\end{array}$ & $\begin{array}{c}63.50 \pm 3.97^{¥} \\
(59.00-68.30)\end{array}$ & $\begin{array}{l}63.10 \pm 3.27 \\
(59.00-66.60)\end{array}$ & $\begin{array}{l}69.47 \pm 6.07 \\
(63.80-78.00)\end{array}$ & $\begin{array}{c}63.35 \pm 0.16^{\mathrm{b}} \\
(63.20-63.50)\end{array}$ \\
\hline $\mathrm{BMI}\left(\mathrm{kg} / \mathrm{m}^{2}\right)$ & $\begin{array}{r}20.47 \pm 1.04^{\mathrm{d}, o} \\
\quad(19.48-22.41)\end{array}$ & $\begin{array}{r}20.49 \pm 1.40^{x_{, \omega}} \\
(18.98-22.59)\end{array}$ & $\begin{array}{l}23.94 \pm 0.93^{*} \\
(22.74-24.88)\end{array}$ & $\begin{array}{r}23.80 \pm 0.84^{\mathrm{a}, \boldsymbol{6}} \\
(22.92-24.88)\end{array}$ & $\begin{array}{l}27.68 \pm 1.92 \\
\quad(25.56-30.47)\end{array}$ & $\begin{array}{l}25.78 \pm 0.48^{b} \\
(25.32-26.23)\end{array}$ \\
\hline Waist (cm) & $\begin{array}{l}67.41 \pm 4.22 \\
(64.50-75.00)^{\circ}\end{array}$ & $\begin{array}{r}67.98 \pm 4.60^{X_{, \omega}} \\
(63.80-75.00)\end{array}$ & $\begin{array}{l}74.25 \pm 5.08 \\
(69.50-79.00)\end{array}$ & $\begin{array}{l}75.33 \pm 4.70 \\
\quad(70.00-81.00)\end{array}$ & $\begin{array}{l}80.17 \pm 8.02 \\
\quad(73.00-91.00)\end{array}$ & $\begin{array}{r}73.75 \pm 2.35^{b} \\
(71.50-76.00)\end{array}$ \\
\hline Hip (cm) & $\begin{array}{r}94.09 \pm 4.57^{\mathrm{d}, 0} \\
(87.00-97.00)\end{array}$ & $\begin{array}{c}93.23 \pm 4.35^{\mathrm{X}, \omega} \\
(87.50-97.00)\end{array}$ & $\begin{array}{c}103.25 \pm 5.61^{¥} \\
(98.00-108.50)\end{array}$ & $\begin{array}{c}101.00 \pm 3.72^{\sigma} \\
(98.00-106.00)\end{array}$ & $\begin{array}{l}107.73 \pm 0.54 \\
(107.00-108.20)\end{array}$ & $\begin{array}{l}106.75 \pm 0.78^{b} \\
(106.00- \\
107.50)\end{array}$ \\
\hline Resistance (Ohm) & $\begin{array}{l}595.89 \pm 85.36 \\
\quad(501.00-735.00)\end{array}$ & $\begin{array}{r}598.33 \pm 69.50^{\omega} \\
(497.00-690.00)\end{array}$ & $\begin{array}{l}576.67 \pm 55.46 \\
\quad(526.00-650.00)\end{array}$ & $\begin{array}{c}567.00 \pm 53.60^{\mathrm{a}} \\
\quad(513.00-636.00)\end{array}$ & $\begin{array}{l}565.13 \pm 35.34 \\
(526.00-611.00)\end{array}$ & $\begin{array}{l}523.00 \pm 24.02^{b} \\
(500.00- \\
546.00)\end{array}$ \\
\hline Reactance (Ohm) & $\begin{array}{c}66.00 \pm 12.24 \\
(56.00-87.00)\end{array}$ & $\begin{array}{l}65.89 \pm 8.46 \\
\quad(56.00-73.00)\end{array}$ & $\begin{array}{c}61.33 \pm 7.74^{*} \\
(53.00-71.00)\end{array}$ & $\begin{array}{r}59.67 \pm 8.75^{\mathrm{a}, 6} \\
\quad(51.00-71.00)\end{array}$ & $\begin{array}{c}73.43 \pm 9.54 \\
(66.00-89.00)\end{array}$ & $\begin{array}{l}69.50 \pm 6.79 \\
(63.00-76.00)\end{array}$ \\
\hline $\mathrm{PA}\left({ }^{\circ}\right)$ & $\begin{array}{r}6.30 \pm 0.35^{\circ} \\
(5.90-6.80)\end{array}$ & $\begin{array}{r}6.29 \pm 0.46^{\omega} \\
(5.70-6.80)\end{array}$ & $\begin{array}{r}6.13 \pm 0.89^{¥} \\
(5.30-7.30)\end{array}$ & $\begin{array}{c}6.03 \pm 0.97^{\mathrm{a}, \sigma} \\
(5.10-7.30)\end{array}$ & $\begin{array}{l}7.40 \pm 0.59 \\
(6.70-8.30)\end{array}$ & $\begin{array}{r}7.55 \pm 0.37^{b} \\
(7.20-7.90)\end{array}$ \\
\hline TBW (L) & $\begin{array}{l}31.80 \pm 2.83 \\
\quad(29.10-36.60)\end{array}$ & $\begin{array}{l}31.66 \pm 3.00 \\
(29.70-36.90)\end{array}$ & $\begin{array}{l}33.13 \pm 2.50^{¥} \\
(31.10-36.50)\end{array}$ & $\begin{array}{c}33.40 \pm 2.56^{\mathrm{a}} \\
\quad(31.10-36.80)\end{array}$ & $\begin{array}{l}32.93 \pm 0.36 \\
\quad(32.60-33.50)\end{array}$ & $\begin{array}{l}33.35 \pm 1.10 \\
(32.30-34.40)\end{array}$ \\
\hline TBW (\%) & $\begin{array}{r}57.92 \pm 4.19^{\natural, o} \\
(50.80-61.30)\end{array}$ & $\begin{array}{l}57.82 \pm 3.19 \\
\quad(52.40-60.40)\end{array}$ & $\begin{array}{l}52.10 \pm 1.37^{*} \\
(50.30-53.40)\end{array}$ & $\begin{array}{l}52.90 \pm 2.01^{\mathrm{a}} \\
\quad(50.60-55.30)\end{array}$ & $\begin{array}{l}47.79 \pm 4.30 \\
\quad(42.00-52.60)\end{array}$ & $\begin{array}{c}52.65 \pm 1.83^{b} \\
(50.90-54.40)\end{array}$ \\
\hline $\mathrm{ECW}(\mathrm{L})$ & $\begin{array}{l}14.11 \pm 1.33 \\
\quad(12.40-16.10)\end{array}$ & $\begin{array}{l}14.07 \pm 1.39 \\
\quad(12.60-16.10)\end{array}$ & $\begin{array}{l}15.10 \pm 2.01^{*} \\
\quad(12.50-17.10)\end{array}$ & $\begin{array}{r}15.37 \pm 2.18^{\mathrm{a}, 6} \\
(12.50-17.40)\end{array}$ & $\begin{array}{l}13.22 \pm 0.74 \\
\quad(12.10-14.00)\end{array}$ & $\begin{array}{r}13.20 \pm 0.84^{b} \\
(12.40-14.00)\end{array}$ \\
\hline ECW (\%) & $\begin{array}{l}44.42 \pm 1.38^{\circ} \\
\quad(42.50-46.00)\end{array}$ & $\begin{array}{l}43.93 \pm 2.64^{\omega} \\
\quad(40.80-47.20)\end{array}$ & $\begin{array}{l}45.53 \pm 3.93^{*} \\
(40.40-49.30)\end{array}$ & $\begin{array}{r}45.87 \pm 4.29^{\mathrm{a}, \mathrm{\sigma}} \\
(40.30-50.10)\end{array}$ & $\begin{array}{l}40.17 \pm 2.02 \\
\quad(37.10-42.60)\end{array}$ & $\begin{array}{l}39.50 \pm 1.36^{\mathrm{b}} \\
(38.20-40.80)\end{array}$ \\
\hline ICW (L) & $\begin{array}{l}17.66 \pm 1.63^{\circ} \\
(16.70-20.50)\end{array}$ & $\begin{array}{c}17.59 \pm 1.86^{\omega} \\
(16.20-20.80)\end{array}$ & $\begin{array}{c}18.00 \pm 1.45^{*} \\
(16.10-19.40)\end{array}$ & $\begin{array}{r}18.03 \pm 1.47^{\mathrm{a}, \sigma} \\
(16.10-19.40)\end{array}$ & $\begin{array}{l}19.71 \pm 0.63 \\
\quad(18.90-20.60)\end{array}$ & $\begin{array}{r}20.15 \pm 0.26^{b} \\
\quad(19.90-20.40)\end{array}$ \\
\hline ICW (\%) & $\begin{array}{l}55.58 \pm 1.38^{\circ} \\
\quad(54.00-57.50)\end{array}$ & $\begin{array}{l}55.62 \pm 1.91^{\omega} \\
\quad(52.92-57.58)\end{array}$ & $\begin{array}{l}54.50 \pm 3.91^{¥} \\
\quad(50.70-59.60)\end{array}$ & $\begin{array}{r}54.12 \pm 4.37^{\mathrm{a}, \mathrm{\sigma}} \\
\quad(49.85-59.81)\end{array}$ & $\begin{array}{l}59.83 \pm 2.02 \\
\quad(57.40-62.90)\end{array}$ & $\begin{array}{c}60.61 \pm 1.37^{b} \\
(59.30-61.92)\end{array}$ \\
\hline TBFat (\%) & $\begin{array}{r}26.55 \pm 2.83^{\mathrm{d}, o} \\
(24.10-29.00)\end{array}$ & - & $\begin{array}{l}35.25 \pm 1.44^{*} \\
(33.90-36.60)\end{array}$ & - & $\begin{array}{l}43.80 \pm 0.00 \\
\quad(43.80-43.80)\end{array}$ & - \\
\hline TBFat (kg) & $\begin{array}{l}15.15 \pm 0.01^{a, o} \\
\quad(15.14-15.16)\end{array}$ & - & $\begin{array}{l}21.73 \pm 1.73^{¥} \\
(20.11-23.34)\end{array}$ & - & $\begin{array}{l}34.10 \pm 0.00 \\
\quad(34.10-34.10)\end{array}$ & - \\
\hline TBLean (kg) & $\begin{array}{l}40.17 \pm 5.98 \\
(34.99-45.35)\end{array}$ & - & $\begin{array}{l}37.61 \pm 0.57 \\
(37.08-38.14)\end{array}$ & - & $\begin{array}{l}41.26 \pm 0.00 \\
\quad(41.26-41.26)\end{array}$ & - \\
\hline $\mathrm{FM}(\mathrm{kg})$ & $\begin{array}{r}11.35 \pm 2.86^{\square, o} \\
(8.10-15.10)\end{array}$ & $\begin{array}{c}11.80 \pm 3.12^{c_{,} X_{, \omega}} \\
(8.10-16.00)\end{array}$ & $\begin{array}{c}18.00 \pm 1.64 \\
\quad(15.90-19.70)\end{array}$ & $\begin{array}{l}17.27 \pm 1.80^{\mathrm{a}} \\
\quad(15.80-19.70)\end{array}$ & $\begin{array}{l}18.65 \pm 0.99 \\
\quad(17.70-19.60)\end{array}$ & $\begin{array}{c}16.95 \pm 0.99^{b} \\
(16.00-17.90)\end{array}$ \\
\hline FM (\%) & $\begin{array}{r}20.30 \pm 4.06^{\mathrm{d}, o} \\
(16.10-26.40)\end{array}$ & $\begin{array}{c}21.05 \pm 4.43^{c, X, \omega} \\
(16.50-27.80)\end{array}$ & $\begin{array}{l}28.37 \pm 2.09 \\
\quad(26.90-31.20)\end{array}$ & $\begin{array}{l}27.57 \pm 2.58^{\mathrm{a}} \\
\quad(25.00-30.90)\end{array}$ & $\begin{array}{l}29.10 \pm 1.36 \\
\quad(27.80-30.40)\end{array}$ & $\begin{array}{r}26.70 \pm 1.46^{\mathrm{b}} \\
(25.30-28.10)\end{array}$ \\
\hline FFM (kg) & $\begin{array}{l}44.23 \pm 3.57 \\
\quad(42.20-50.00)\end{array}$ & $\begin{array}{l}43.75 \pm 4.06 \\
\quad(41.10-50.30)\end{array}$ & $\begin{array}{l}45.50 \pm 3.25 \\
\quad(43.10-49.90)\end{array}$ & $\begin{array}{l}45.83 \pm 3.32^{\mathrm{a}} \\
\quad(43.20-50.30)\end{array}$ & $\begin{array}{l}45.50 \pm 0.63 \\
\quad(44.90-46.10)\end{array}$ & $\begin{array}{r}46.40 \pm 0.84^{b} \\
(45.60-47.20)\end{array}$ \\
\hline FFM (\%) & $\begin{array}{r}79.70 \pm 4.06^{\mathrm{d}, o} \\
(73.60-83.90)\end{array}$ & $\begin{array}{c}78.95 \pm 4.43^{c, X_{,} \omega} \\
(72.20-83.50)\end{array}$ & $\begin{array}{l}71.63 \pm 2.09 \\
(68.80-73.10)\end{array}$ & $\begin{array}{l}72.43 \pm 2.58^{\mathrm{a}} \\
\quad(69.10-75.00)\end{array}$ & $\begin{array}{l}70.90 \pm 1.36 \\
(69.60-72.20)\end{array}$ & $\begin{array}{c}73.30 \pm 1.46^{\mathrm{b}} \\
(71.90-74.70)\end{array}$ \\
\hline
\end{tabular}

Results are expressed in mean value \pm standard deviation, and minimum and maximum for each parameter. Values of $\mathrm{p}<0.05$ are considered significant

$B M I$ body mass index, ECW extracellular water, FM fat mass, FFM fat free mass, ICW intracellular water, NWL normal weight lean, NWO normal weight obese, PA phase angle, $P r e O B / O B$ preobese-obese, $T B$ total body, $T B W$ total body water

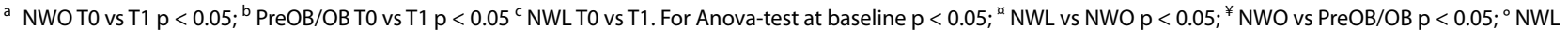
vs PreOB/OB $p<0.05$. Anova-test after POS $p<0.05 ;{ }^{\gamma}$ NWL vs NWO $p<0.05 ;{ }^{6}$ NWO vs PreOB/OB $p<0.05 ;{ }^{\omega}$ NWL vs PreOB/OB $p<0.05$ 
Table 2 Comparison of psychometric parameters of normal weight lean, normal weight obese and pre-obese/obese groups of women between baseline and after 3 weeks POS treatment

\begin{tabular}{|c|c|c|c|c|c|c|}
\hline & \multicolumn{2}{|l|}{ NWL } & \multicolumn{2}{|l|}{ NWO } & \multicolumn{2}{|l|}{ PreobOB } \\
\hline & Baseline & POS & Baseline & POS & Baseline & POS \\
\hline EDI-2_DT & $\begin{array}{c}2.89 \pm 4.63 \\
(0.00-20.00)\end{array}$ & $\begin{array}{l}2.94 \pm 4.40 \\
(0.00-19.00)\end{array}$ & $\begin{array}{l}5.26 \pm 6.33 \\
(0.00-21.00)\end{array}$ & $\begin{array}{l}5.09 \pm 6.08 \\
(0.00-20.00)\end{array}$ & $\begin{array}{l}8.41 \pm 7.58 \\
(0.00-18.00)\end{array}$ & $\begin{array}{c}8.50 \pm 7.84 \\
(0.00-19.00)\end{array}$ \\
\hline EDI-2_B & $\begin{array}{l}1.00 \pm 2.45 \\
(0.00-10.00)\end{array}$ & $\begin{array}{c}0.39 \pm 1.24 \\
(0.00-5.00)\end{array}$ & $\begin{array}{c}0.91 \pm 1.64 \\
(0.00-7.00)\end{array}$ & $\begin{array}{r}0.53 \pm 1.16^{\mathrm{a}} \\
(0.00-5.00)\end{array}$ & $\begin{array}{c}3.64 \pm 4.12 \\
(0.00-16.00)\end{array}$ & $\begin{array}{l}2.50 \pm 2.89^{b} \\
(0.00-10.00)\end{array}$ \\
\hline EDI-2_BD & $\begin{array}{l}5.28 \pm 6.09 \\
(0.00-23.00)\end{array}$ & $\begin{array}{l}4.94 \pm 6.01 \\
(0.00-23.00)\end{array}$ & $\begin{array}{l}9.29 \pm 7.81 \\
(0.00-27.00)\end{array}$ & $\begin{array}{c}7.50 \pm 7.18^{\mathrm{a}} \\
(0.00-25.00)\end{array}$ & $\begin{array}{c}14.09 \pm 6.35 \\
(2.00-24.00)\end{array}$ & $\begin{array}{r}11.91 \pm 5.76^{b} \\
(0.00-20.00)\end{array}$ \\
\hline EDI-2_I & $\begin{array}{c}1.39 \pm 2.20 \\
(0.00-6.00)\end{array}$ & $\begin{array}{c}1.22 \pm 1.90 \\
(0.00-5.00)\end{array}$ & $\begin{array}{c}3.26 \pm 4.29 \\
(0.00-17.00)\end{array}$ & $\begin{array}{c}1.62 \pm 2.85^{\mathrm{a}} \\
(0.00-10.00)\end{array}$ & $\begin{array}{l}5.82 \pm 4.92 \\
(1.00-19.00)\end{array}$ & $\begin{array}{c}3.68 \pm 4.51^{b} \\
(0.00-16.00)\end{array}$ \\
\hline EDI-2_P & $\begin{array}{l}3.83 \pm 3.54 \\
(0.00-14.00)\end{array}$ & $\begin{array}{c}3.61 \pm 3.16 \\
(0.00-13.00)\end{array}$ & $\begin{array}{l}4.85 \pm 3.00 \\
(0.00-11.00)\end{array}$ & $\begin{array}{l}4.74 \pm 2.99 \\
(0.00-12.00)\end{array}$ & $\begin{array}{l}3.36 \pm 3.67 \\
(0.00-14.00)\end{array}$ & $\begin{array}{l}3.32 \pm 3.81 \\
(0.00-15.00)\end{array}$ \\
\hline EDI-2_ID & $\begin{array}{l}2.33 \pm 2.47 \\
(0.00-9.00)\end{array}$ & $\begin{array}{c}2.06 \pm 2.39 \\
(0.00-9.00)\end{array}$ & $\begin{array}{l}3.53 \pm 3.55 \\
(0.00-16.00)\end{array}$ & $\begin{array}{l}3.47 \pm 3.67 \\
(0.00-17.00)\end{array}$ & $\begin{array}{l}3.68 \pm 3.37 \\
(0.00-10.00)\end{array}$ & $\begin{array}{l}3.59 \pm 3.45 \\
(0.00-10.00)\end{array}$ \\
\hline EDI-2_IA & $\begin{array}{l}3.61 \pm 3.96 \\
\quad(0.00-12.00)\end{array}$ & $\begin{array}{l}3.22 \pm 3.61 \\
\quad(0.00-11.00)\end{array}$ & $\begin{array}{l}4.47 \pm 5.93 \\
(0.00-23.00)\end{array}$ & $\begin{array}{l}4.41 \pm 6.03 \\
(0.00-24.00)\end{array}$ & $\begin{array}{l}5.36 \pm 5.02 \\
(0.00-16.00)\end{array}$ & $\begin{array}{l}5.27 \pm 4.73 \\
\quad(0.00-15.00)\end{array}$ \\
\hline EDI-2_MF & $\begin{array}{l}7.89 \pm 5.14 \\
(1.00-21.00)\end{array}$ & $\begin{array}{l}7.67 \pm 4.98 \\
(1.00-20.00)\end{array}$ & $\begin{array}{l}5.76 \pm 3.24 \\
\quad(0.00-14.00)\end{array}$ & $\begin{array}{l}5.59 \pm 3.28 \\
(0.00-15.00)\end{array}$ & $\begin{array}{l}5.50 \pm 4.59 \\
\quad(1.00-20.00)\end{array}$ & $\begin{array}{l}5.45 \pm 4.27 \\
\quad(1.00-19.00)\end{array}$ \\
\hline EDI-2_A & $\begin{array}{c}3.50 \pm 2.46 \\
(0.00-8.00)\end{array}$ & $\begin{array}{c}3.56 \pm 2.50 \\
(0.00-9.00)\end{array}$ & $\begin{array}{c}3.53 \pm 1.97 \\
(0.00-7.00)\end{array}$ & $\begin{array}{l}3.38 \pm 1.97 \\
(0.00-8.00)\end{array}$ & $\begin{array}{c}4.50 \pm 2.09 \\
(1.00-8.00)\end{array}$ & $\begin{array}{c}4.27 \pm 2.07 \\
(0.00-7.00)\end{array}$ \\
\hline EDI-2_IR & $\begin{array}{c}1.61 \pm 2.48 \\
(0.00-9.00)\end{array}$ & $\begin{array}{r}1.67 \pm 2.28 \\
(0.00-8.00)\end{array}$ & $\begin{array}{l}3.50 \pm 3.86 \\
(0.00-14.00)\end{array}$ & $\begin{array}{l}3.68 \pm 4.09 \\
(0.00-15.00)\end{array}$ & $\begin{array}{c}2.32 \pm 2.92 \\
(0.00-9.00)\end{array}$ & $\begin{array}{l}2.27 \pm 2.81 \\
(0.00-9.00)\end{array}$ \\
\hline EDI-2_SI & $\begin{array}{c}2.39 \pm 2.17 \\
(0.00-7.00)\end{array}$ & $\begin{array}{r}2.33 \pm 2.00 \\
(0.00-6.00)\end{array}$ & $\begin{array}{l}4.03 \pm 3.38 \\
(0.00-15.00)\end{array}$ & $\begin{array}{l}3.94 \pm 3.20 \\
\quad(0.00-14.00)\end{array}$ & $\begin{array}{l}4.00 \pm 3.41 \\
(0.00-14.00)\end{array}$ & $\begin{array}{l}3.91 \pm 3.38 \\
(0.00-15.00)\end{array}$ \\
\hline SCL90R_Som & $\begin{array}{c}0.67 \pm 0.12 \\
(0.50-0.83)\end{array}$ & $\begin{array}{r}0.30 \pm 0.15^{c} \\
(0.08-0.50)\end{array}$ & $\begin{array}{c}0.61 \pm 0.35 \\
(0.33-1.08)\end{array}$ & $\begin{array}{r}0.28 \pm 0.11^{\mathrm{a}} \\
(0.17-0.42)\end{array}$ & $\begin{array}{c}0.87 \pm 0.63 \\
(0.08-1.67)\end{array}$ & $\begin{array}{r}0.42 \pm 0.26^{b} \\
(0.17-0.67)\end{array}$ \\
\hline SCL90R_Obs Comp & $\begin{array}{c}0.81 \pm 0.36 \\
(0.50-1.30)\end{array}$ & $\begin{array}{r}0.37 \pm 0.13^{c} \\
(0.30-0.60)\end{array}$ & $\begin{array}{c}0.60 \pm 0.23 \\
(0.30-0.80)\end{array}$ & $\begin{array}{r}0.27 \pm 0.10^{\mathrm{a}} \\
(0.20-0.40)\end{array}$ & $\begin{array}{c}0.46 \pm 0.34 \\
(0.20-1.00)\end{array}$ & $\begin{array}{r}0.20 \pm 0.00^{b} \\
(0.20-0.20)\end{array}$ \\
\hline SCL90R_Interp Sens & $\begin{array}{c}0.75 \pm 0.41 \\
(0.22-1.33)\end{array}$ & $\begin{array}{r}0.22 \pm 0.23^{c} \\
(0.00-0.56)\end{array}$ & $\begin{array}{c}0.56 \pm 0.09 \\
(0.44-0.67)\end{array}$ & $\begin{array}{r}0.26 \pm 0.11^{\mathrm{a}} \\
(0.11-0.33)\end{array}$ & $\begin{array}{c}0.45 \pm 0.29 \\
(0.11-0.89)\end{array}$ & $\begin{array}{r}0.56 \pm 0.00^{b} \\
(0.56-0.56)\end{array}$ \\
\hline SCL90R_Dep & $\begin{array}{c}0.70 \pm 0.35 \\
\quad(0.38-1.31)\end{array}$ & $\begin{array}{r}0.27 \pm 0.26^{c} \\
(0.00-0.69)\end{array}$ & $\begin{array}{l}0.18 \pm 0.04 \\
\quad(0.15-0.23)\end{array}$ & $\begin{array}{l}0.26 \pm 0.10 \\
(0.15-0.38)\end{array}$ & $\begin{array}{l}0.52 \pm 0.67 \\
\quad(0.08-1.62)\end{array}$ & $\begin{array}{c}0.23 \pm 0.24 \\
(0.00-0.46)\end{array}$ \\
\hline SCL90R_Anx & $\begin{array}{c}0.56 \pm 0.34 \\
(0.12-1.00)\end{array}$ & $\begin{array}{c}0.17 \pm 0.15 \\
\quad(0.10-0.43)\end{array}$ & $\begin{array}{l}0.30 \pm 0.22 \\
(0.04-0.57)\end{array}$ & $\begin{array}{l}0.31 \pm 0.14 \\
(0.21-0.50)\end{array}$ & $\begin{array}{c}0.66 \pm 0.42 \\
(0.03-1.19)\end{array}$ & $\begin{array}{r}0.08 \pm 0.08^{b} \\
(0.00-0.16)\end{array}$ \\
\hline SCL90R_Anger Host & $\begin{array}{c}0.50 \pm 0.22 \\
(0.17-0.67)\end{array}$ & $\begin{array}{r}0.20 \pm 0.07^{c} \\
(0.17-0.33)\end{array}$ & $\begin{array}{c}0.39 \pm 0.16 \\
(0.17-0.50)\end{array}$ & $\begin{array}{r}0.17 \pm 0.14^{\mathrm{a}} \\
(0.00-0.33)\end{array}$ & $\begin{array}{c}0.78 \pm 0.49 \\
(0.00-1.33)\end{array}$ & $\begin{array}{r}0.17 \pm 0.17^{b} \\
(0.00-0.33)\end{array}$ \\
\hline SCL90R_Phob & $\begin{array}{l}0.10 \pm 0.17 \\
(0.00-0.43)\end{array}$ & $\begin{array}{c}0.06 \pm 0.13 \\
(0.00-0.29)\end{array}$ & $\begin{array}{l}0.33 \pm 0.19 \\
(0.14-0.57)\end{array}$ & $\begin{array}{r}0.19 \pm 0.07^{\mathrm{a}} \\
(0.14-0.29)\end{array}$ & $\begin{array}{c}0.37 \pm 0.56 \\
(0.00-1.29)\end{array}$ & $\begin{array}{r}0.07 \pm 0.07^{b} \\
(0.00-0.14)\end{array}$ \\
\hline SCL90R_Paran & $\begin{array}{c}0.82 \pm 0.63 \\
(0.00-1.67)\end{array}$ & $\begin{array}{r}0.31 \pm 0.27^{c} \\
(0.00-0.67)\end{array}$ & $\begin{array}{c}0.17 \pm 0.25 \\
(0.00-0.50)\end{array}$ & $\begin{array}{r}0.00 \pm 0.00^{\mathrm{a}} \\
(0.00-0.00)\end{array}$ & $\begin{array}{c}0.39 \pm 0.32 \\
(0.00-0.83)\end{array}$ & $\begin{array}{r}0.17 \pm 0.17^{b} \\
(0.00-0.33)\end{array}$ \\
\hline SCL90R_Psych & $\begin{array}{c}0.26 \pm 0.18 \\
(0.10-0.60)\end{array}$ & $\begin{array}{r}0.06 \pm 0.08 \\
(0.00-0.18)\end{array}$ & $\begin{array}{l}0.26 \pm 0.17 \\
\quad(0.11-0.49)\end{array}$ & $\begin{array}{r}0.15 \pm 0.13^{\mathrm{a}} \\
(0.00-0.31)\end{array}$ & $\begin{array}{c}0.34 \pm 0.40 \\
(0.00-0.98)\end{array}$ & $\begin{array}{r}0.08 \pm 0.08^{b} \\
(0.00-0.16)\end{array}$ \\
\hline SCL90R_GSI & $\begin{array}{c}0.58 \pm 0.23 \\
(0.29-0.92)\end{array}$ & $\begin{array}{r}0.23 \pm 0.07^{c} \\
(0.17-0.32)\end{array}$ & $\begin{array}{c}0.45 \pm 0.20 \\
(0.23-0.69)\end{array}$ & $\begin{array}{c}0.25 \pm 0.02^{\mathrm{a}} \\
(0.22-0.27)\end{array}$ & $\begin{array}{c}0.54 \pm 0.38 \\
(0.14-1.13)\end{array}$ & $\begin{array}{r}0.24 \pm 0.12^{b} \\
(0.13-0.36)\end{array}$ \\
\hline BUT_GSI & $\begin{array}{l}0.55 \pm 0.43 \\
(0.09-1.21)\end{array}$ & $\begin{array}{r}0.39 \pm 0.43^{c} \\
(0.12-1.15)\end{array}$ & $\begin{array}{l}1.11 \pm 0.66 \\
(0.24-1.71)\end{array}$ & $\begin{array}{l}0.79 \pm 0.64 \\
\quad(0.15-1.62)\end{array}$ & $\begin{array}{c}1.08 \pm 0.69 \\
(0.41-2.09)\end{array}$ & $\begin{array}{r}0.54 \pm 0.41^{b} \\
(0.15-0.94)\end{array}$ \\
\hline BUT_WP & $\begin{array}{c}0.93 \pm 0.82 \\
(0.13-2.38)\end{array}$ & $\begin{array}{r}0.79 \pm 1.05 \\
(0.13-2.63)\end{array}$ & $\begin{array}{l}1.96 \pm 1.17 \\
(0.38-2.88)\end{array}$ & $\begin{array}{l}1.42 \pm 1.27 \\
(0.38-3.13)\end{array}$ & $\begin{array}{c}1.97 \pm 0.60 \\
(1.13-2.75)\end{array}$ & $\begin{array}{r}1.13 \pm 0.65^{b} \\
(0.50-1.75)\end{array}$ \\
\hline BUT_BIC & $\begin{array}{c}0.58 \pm 0.62 \\
(0.11-1.56)\end{array}$ & $\begin{array}{r}0.37 \pm 0.56^{c} \\
(0.00-1.33)\end{array}$ & $\begin{array}{c}1.48 \pm 0.85 \\
(0.44-2.44)\end{array}$ & $\begin{array}{c}1.00 \pm 0.78 \\
(0.22-2.00)\end{array}$ & $\begin{array}{c}1.27 \pm 1.20 \\
(0.11-3.00)\end{array}$ & $\begin{array}{r}0.67 \pm 0.58^{b} \\
(0.11-1.22)\end{array}$ \\
\hline BUT_A & $\begin{array}{c}0.18 \pm 0.20 \\
(0.00-0.50)\end{array}$ & $\begin{array}{r}0.04 \pm 0.07^{c} \\
(0.00-0.17)\end{array}$ & $\begin{array}{l}0.28 \pm 0.22 \\
(0.00-0.50)\end{array}$ & $\begin{array}{r}0.11 \pm 0.16^{\mathrm{a}} \\
(0.00-0.33)\end{array}$ & $\begin{array}{c}0.13 \pm 0.14 \\
(0.00-0.33)\end{array}$ & $\begin{array}{r}0.17 \pm 0.17^{b} \\
(0.00-0.33)\end{array}$ \\
\hline BUT_CSM & $\begin{array}{c}0.71 \pm 0.48 \\
(0.00-1.33)\end{array}$ & $\begin{array}{r}0.57 \pm 0.30 \\
(0.17-1.00)\end{array}$ & $\begin{array}{c}1.06 \pm 0.67 \\
(0.17-1.67)\end{array}$ & $\begin{array}{l}0.89 \pm 0.87 \\
(0.00-2.00)\end{array}$ & $\begin{array}{c}0.99 \pm 0.73 \\
(0.17-1.83)\end{array}$ & $\begin{array}{r}0.33 \pm 0.35^{b} \\
(0.00-0.67)\end{array}$ \\
\hline
\end{tabular}


Table 2 continued

\begin{tabular}{|c|c|c|c|c|c|c|}
\hline & \multicolumn{2}{|l|}{ NWL } & \multicolumn{2}{|l|}{ NWO } & \multicolumn{2}{|l|}{ PreobOB } \\
\hline & Baseline & POS & Baseline & POS & Baseline & POS \\
\hline$\overline{\text { BUT_D }}$ & $\begin{array}{l}0.13 \pm 0.10 \\
(0.00-0.20)\end{array}$ & $\begin{array}{r}0.00 \pm 0.00^{c} \\
(0.00-0.00)\end{array}$ & $\begin{array}{r}0.13 \pm 0.20 \\
(0.00-0.40)\end{array}$ & $\begin{array}{r}0.13 \pm 0.20 \\
(0.00-0.40)\end{array}$ & $\begin{array}{c}0.57 \pm 0.75 \\
(0.00-1.80)\end{array}$ & $\begin{array}{r}0.10 \pm 0.10^{b} \\
(0.00-0.20)\end{array}$ \\
\hline
\end{tabular}

Results are expressed in mean value \pm standard deviation, and minimum and maximum for each parameter. Values of $\mathrm{p}<0.05$ are considered significant

$A$ asceticism, $A$ avoidance, Anger Host anger/hostility, $A n x$ anxious, $B$ bulimia, $B D$ body dissatisfaction, $B I C$ body image concerns, $B M I$ body mass index, $B U T$ body uneasiness test, CSM compulsive self-monitoring, $D$ depersonalization, Dep depression, $D T$ drive for thinness, EDI-2 eating disorder inventory-2, GSI global severity index, I ineffectiveness, IA interoceptive awareness, IC body image, ID interpersonal distrust, IG intervention group, Interp Sens interpersonal sensitivity, IR impulse regulation, MF maturity fears, NWL normal weight lean, NWO normal weight obese, Obs obsessive/compulsive, $P$ perfectionism, $P a r a n$ paranoia, $P$ hob phobia, $P O S$ probiotic oral suspension, $P r e O B / O B$ preobese-obese, $P S D I$ positive symptom distress index, PST positive symptom total, Psych psychoticism, SCL90R symptom checklist 90, S/ social insecurity, Som somatization, WP weight phobia

${ }^{\text {a }}$ NWO T0 vs T1 $\mathrm{p}<0.05 ;{ }^{\mathrm{b}}$ PreOB/OB T0 vs T1 $\mathrm{p}<0.05 ;{ }^{\mathrm{c}} \mathrm{NWL}$ T0 vs T1

$3.64 \pm 4.12 ; 3$ weeks POS, $2.50 \pm 2.89)$, the $-15.48 \Delta \%$ of DT (baseline, $14.09 \pm 6.35$; 3 weeks POS, $11.91 \pm 5.76$ ), the $-36.72 \Delta \%$ of I (baseline, $5.82 \pm 4.92 ; 3$ weeks POS, $3.68 \pm 4.51$ ) (Table 2).

A significant improvement of the orocaecal transit time and gastrointestinal symptoms were observed $(\mathrm{p}<0.05)$ after 3 weeks of POS treatment respect to placebo. Moreover, significant differences were observed for meteorism $(\mathrm{p}<0.05)$ and defecation frequency $(\mathrm{p}<0.05)$ (data not show).

\section{Discussion}

The link between the "somatic" and "mental" is undeniably a subject that has fascinated through the years and until the present day many artists and philosophers. Also researchers and scientists daily make their contribution to reveal this chimera showing a complex but fascinating harmonic unit.

The gut-microbiota-brain axis includes the central nervous system, neuroendocrine and neuro-immune system, the sympathetic and parasympathetic arms of the autonomic nervous system, the enteric nervous system and, most importantly, the intestinal microbiota [38]. Some bacteria within the human gastrointestinal tract have the capacity to produce many neurotransmitters and neuromodulators, such as norepinephrine, serotonin, dopamine, acetylcholine, histamine and gamma-aminobutyric acid [7]. Due to these new evidences about the fundamental role of gut microbiota in the alteration of immune, neural and endocrine pathways, the so-called "gut-brain axis" is acquiring new significance, even if the communication routes are not yet defined [38].

From clinical experience and from the literature it is clear the importance of "personalization" of treatment, also respect to the individuals we have in care, taking into account body composition, gut microbiota, feeding behaviour, attitude towards food and the presence of emotional states that may influence them. The intake of probiotic influences the secretion of molecules on which depend anxiety and depression, and simultaneously affects neuroendocrine response to stress.

Recent data show the strong correlation between dysbiosis and a variety of conditions such as obesity, allergies, autoimmune disorders, Irritable Bowel Syndrome, Inflammatory Bowel Disease and psychiatric disorders [39, 40]. Bifidobacterium and Atopobium were significantly less abundant in obese animals compared to the non-obese rats, in conjunction with significantly higher levels of the Clostridium cluster XIVa and Lactobacillus group [41]. In the meantime, Cani et al. reported a reduction in the Clostridium cluster XIVa (Clostridium coccoides) group, along with lower Bifidobacterium and Bacteroides levels in mice fed high-fat diet. An increase of Firmicutes levels was observed in high-fat fed mice, while Bacteroides phylum decreased overtime in obese animals [42]. Angelakis et al. [43], highlighted elevated levels of Bacteroidetes phylum, a strong abundance of the Firmicutes phylum and elevated concentrations of Lactobacilli in the gut microbiota of obese and overweight adults compared to lean individuals. Moreover, the supplementation with lactic acid bacteria brought to weight modification, suppression of the neuroendocrine stress response and relieved abdominal dysfunction [44, 45].

Consistent with these considerations, it has been prepared a new formulation of POS, containing different strain of bacteria, i.e. Streptococcus thermophiles, Bifidobacterium animalis subsp. Lactis, Streptococcus thermophiles, Lactobacillus bulgaricus, Lactococcus lactis subsp. Lactis, Lactobacillus acidophilus, Lactobacillus plantarum, Lactobacillus reuteri, for a total of $120 \times 10^{10}$ colony-forming unit CFU.

We tested the effectiveness of this new POS formulation, having two main purposes: (1) to investigate the correlation between body composition and the presence of psychological disorders and psychopathological symptoms in people affected by NWO syndrome and obesity 
respect to normal lean individual; (2) to check whether the intake of POS could change all the examined parameters, in order to make an early diagnosis and to block any nascent development of a psychopathological disorder, taking into account all its future consequences.

In the present study, the cut-off point of total body fat was 30\% [46]; the analysis of anthropometric and body composition values showed $24 \%$ of NWO, $26 \%$ of NWL and $50 \%$ of PreOB/OB women. In particular, between the NWL versus the NWO group, were highlighted several significant differences $(\mathrm{p}<0.05)$, like weight, BMI, hip circumference, TBW (\%), TBFat (\% and g), FM (kg and $\%$ ) and FFM (\%), as well as in NWL versus the PreOB/OB group, in terms of weight, BMI, waist and hip circumference, PA $\left(^{\circ}\right)$, TBW (\%), ECW (\%), ICW (L and \%), TBFat (\% and g), FM (kg and \%) and FFM (\%) ( $<<0.05)$.

Moreover, significant differences $(\mathrm{p}<0.05)$ between the NWO and the PreOB/OB groups in terms of weight, BMI, Reactance, PA, TBW (L and \%), ECW (L and \%), ICW (L and \%), TBFat (\% and g) were observed.

As a challenge in the development of effective strategies to prevent the increased prevalence of obesity, data reported in this study highlight the efficacy of the POS treatment on body composition in subjects with more than $30 \%$ of total body fat, given the considerable improvement of BMI, FM ( $\mathrm{kg}$ and \%), hydration status as TBW, ECW and ICW, and FFM $(\mathrm{p}<0.05)$ in NWO group, as well as the improvement of weight, BMI, waist, hip, resistance, PA, TBW, ICW ECW, FM, and FFM in the PreobOB group $(\mathrm{p}<0.05)$, suggesting a safe and effective intervention for general population, with substantial benefits to public health.

Many neurotransmitters and neuromodulators secreted by bacteria are able to modulate the state of the host mood: gamma-aminobutyric acid is produced by certain Lactobacillus and Bifidobacterium species; norepinephrine is released by Escherichia, Bacillus and Saccharomyces spp.; 5 Hydroxy Triptamine is released by Candida, Streptococcus, Escherichia and Enterococcus spp.; dopamine is produced by Bacillus and acetylcholine by Lactobacillus [47]. Dinan et al. have reported that probiotic Bifidobacterium infantis 35624 has shown to have an antidepressant action in preclinical models of depression acting as a psychobiotic with a mental health benefit [48]. Gut composition is affected also by the resilience to environmental stress, impairing the cortisol awaking response and emotional reaction in healthy subjects [49]. On the other hand, it has been shown that psychological stress itself leads to dysbiosis [50, 51], turning in a vicious circle.

Our results seem to confirm the high prevalence of body image disorders in NWO and PreoOB/OB patients. According to literature data, we provided the evidence that POS therapy improves the psychological state, reducing the positivity to BUT $(-24.90 \%)$ and the alteration of body image perception, as demonstrated by the significant reduction in the subscales of the EDI-2 responses both in NWO than in PreOB/OB $(-41.94 \Delta \%$ of $\mathrm{B}$, the $-19.30 \Delta \%$ of DT, the $-50.45 \Delta \%$ of I in NWO; $-31.25 \Delta \%$ of B, the $-15.48 \Delta \%$ of DT in PreOB/OB).

It has been demonstrated that the oral administration of the probiotic Bifidobacterium longum NCC3001 (Morinaga, Japan) is able to prevent the anxiety-like behavior associated with gut inflammation in animals with an intact vagus nerve [51], as did Lactobacillus rhamnosus and Lactobacillus hilgardii [52]. Lactobacillus reuteri prevented the physiological signs of visceral pain, with a reduction in cardio-autonomic response [53], and Bifidobacterium lactis decreased visceral hypersensitivity when colorectal distention occurred in the context of psychological stress.

Hence, the lactulose passes intact the stomach and small intestine, and reaches the caecum, where the bacteria (normally present in the colon flora) break it down, leading to the production of hydrogen. Part of the hydrogen that forms is absorbed by the intestinal mucous and therefore enters the bloodstream before being released at the pulmonary alveoli and expired. By evaluating the time at which hydrogen appears in the breath, we are indirectly able to determine orocaecal transit time [35]. After POS treatment, a reduction of bacterial overgrowth $(\mathrm{p}<0.05)$ was observed in NWO and PreOB/OB when compared to controls. After POS therapy, a statistically significant improvement was seen in the intestinal transit time in all subjects. An increase in the number of weekly defecations and a reduction of meteorism in subjects affected by constipation were recorded.

\section{Conclusions}

A 3-week intake of selected probiotics, by modulating body composition, bacterial contamination, psychopathological scores and eating behaviour of women affected by NWO syndrome and obesity, offers a tractable approach to problems related to obesity, psychological state and unhealthy eating. One improvement of this study may be to extend the administration period of POS, as probiotics need several weeks to proliferate.

Despite the limitations of our study, related to the study design and the low sample size, our results highlighted that this new formulation of POS may possibly have potential as a therapeutic strategy for prevention and/ or treatment of certain eating behaviour disorders and anxiety-like conditions, to avoid a worsening of the psychiatric symptomatology, the establishment of a global functional impairment of the subject and to improve the 
quality of life of patients. Moreover, these results highlight the need for a more detailed psychiatric evaluation of subjects with an alteration of body image perception, even when this alteration does not fit into a previous pattern referred as eating disorder.

Further research is needed on a larger population and for a longer period of treatment with a controlled trial before definitive conclusions can be made.

\begin{abstract}
Abbreviations
A: asceticism; A: avoidance; Anger Host: anger/hostility; Anx: anxious; B: bulimia; BCM: body cell mass; BCMI: body cell mass index; BD: body dissatisfaction; BIC: body image concerns; BMI: body mass index; BUT: body uneasiness test; CFU: colony-forming unit; CG: control group; CSM: compulsive selfmonitoring; D: depersonalization; Dep: depression; DT: drive for thinness; DXA: dual X-ray absorptiometry; ECW: extracellular water; EDI-2: eating disorder inventory-2; Gl: gastrointestinal; GSI: global severity index; I: ineffectiveness; IA: interoceptive awareness; IC: body image; ICW: intracellular water; ID: interpersonal distrust; IG: intervention group; Interp Sens: interpersonal sensitivity; IR: impulse regulation; LBT: lactulose breath test; LPL: lipoprotein lipase; LPS: lipopolysaccharide; MF: maturity fears; NS: not significant; NWL: normal weight lean; NWO: normal weight obese; Obs: obsessive/compulsive; P: perfectionism; PA: phase angle; Paran: paranoia; PBF: body fat percentage; Phob: phobia; POS: probiotic oral suspension; PreOB/OB: preobese-obese; PSDI: positive symptom distress index; PST: positive symptom total; Psych: psychoticism; SCL90R: symptom checklist 90; SI: social insecurity; Som: somatization; TBBone: total body bone; TBFat: total body fat; TBLean: total body lean; TBW: total body water; WP: weight phobia.
\end{abstract}

\section{Authors' contributions}

The authors'responsibilities were as follows: ADL had primary responsibility for the final content; SG, GM, GC, MM: performed the experiments, and collected the data; $P G$, analyzed the data; $L D R$ drafted the manuscript, and designed the study. ADL, MC, GM, PG, GC, MM, DV, RD, LDR contributed to the interpretation of the data and revision of the manuscript. All authors read and approved the final manuscript.

\section{Author details}

${ }^{1}$ Section of Clinical Nutrition and Nutrigenomic, Department of Biomedicine and Prevention, University of Rome Tor Vergata, 00133 Rome, Italy. ${ }^{2}$ Department of Experimental Medicine and Surgery, University of Rome "Tor Vergata", Rome, Italy. ${ }^{3}$ Emergency Department, "A. Gemelli" General Hospital Foundation, Catholic University of Sacred Heart, 00168 Rome, Italy. ${ }^{4}$ PhD School of Applied Medical-Surgical Sciences, University of Rome "Tor Vergata", 00133 Rome, Italy. ${ }^{5}$ USL 1 UmbriaCastiglione del Lago, 06061 Perugia, Italy. ${ }^{6}$ Department of Surgical Sciences, University Hospital "Umberto I", "Sapienza" University of Rome, 00161 Rome, Italy. ${ }^{7}$ Department of Experimental Medicine and Surgery, University of Rome Tor Vergata, 00133 Rome, Italy. ${ }^{8}$ Section of Clinical Nutrition and Nutrigenomic, Department of Biomedicine and Prevention, University of Rome Tor Vergata, Via Montpellier 1, 00136 Rome, Italy.

\section{Acknowledgements}

We are indebted to all the subjects who volunteered in the study. We also thank the entire medical team from the Section of Clinical Nutrition and Nutrigenomic, University of Rome Tor Vergata, Rome, Emergency Department, "A. Gemelli" General Hospital Foundation, Catholic University of Sacred Heart, and Nuova Annunziatella" Clinic for their technical assistance in conducting the clinical aspects of this study. This study was supported by grants from Ministry of Agriculture, Food and Forestry (D.M.; 2017188).

\section{Competing interests}

The authors declare that they have no competing interests.

\section{Availability of data and materials}

The datasets used and/or analyzed during the current study are available from the corresponding author on reasonable request.

\section{Ethics approval and consent to participate}

This study was approved by the Institutional Review Board of University of Rome "Tor Vergata", Nuova Annunziatella" Clinic and General Hospital Foundation, Catholic University of Sacred Heart.

Study design was clearly written in lay person language and provided to each study subject. All participants recruited in the study authorized their participation by reading and signing the informed consent, conducted in accordance with the provisions of the ethics committee and with the Helsinki Declaration of 1975 as revised in 1983. The participants received no financial compensation or gifts.

\section{Funding}

Supported by grants from Ministry of Agriculture, Food and Forestry (D.M.: 2017188 03/24/2011)

\section{Publisher's Note}

Springer Nature remains neutral with regard to jurisdictional claims in published maps and institutional affiliations.

Received: 24 March 2017 Accepted: 1 June 2017

Published online: 10 June 2017

\section{References}

1. Dethlefsen L, Eckburg PB, Bik EM, Relman DA. Assembly of the human intestinal microbiota. Trends Ecol Evol. 2006;21:517-23.

2. Dinan TG, Stanton C, Cryan JF. Psychobiotics: a novel class of psychotropic. Biol Psychiatry. 2013;74(10):720-6. doi:10.1016/j. biopsych.2013.05.001.

3. Diaz Heijtz R, Wang S, Anuar F, Qian Y, Bjorkholm B, Samuelsson A, et al. Normal gut microbiota modulates brain development and behavior. Proc Natl Acad Sci USA. 2011:108(7):3047-52.

4. Neufeld KA, Kang N, Bienenstock J, Foster JA. Effects of intestinal microbiota on anxiety-like behavior. Commun Integr Biol. 2011:4(4):492-4.

5. Cryan JF, Dinan TG. Mind-altering microorganisms: the impact of the gut microbiota on brain and behaviour. Nat Rev Neurosci. 2012;13(10):701-12

6. Liu YW, Su YW, Ong WK, Cheng TH, Tsai YC. Oral administration of Lactobacillus plantarum K68 ameliorates DSS induced ulcerative colitis in $\mathrm{BALB} / \mathrm{C}$ mice via the anti-inflammatory and immunomodulatory activities. Int Immunopharmacol. 2011;11:2159-66.

7. Moloney RD, Desbonnet L, Clarke G, Dinan TG, Cryan JF. The microbiome: stress, health and disease. Mamm Genome. 2014;25:49-74.

8. Mangiola F, laniro G, Franceschi F, Fagiuoli S, Gasbarrini G, Gasbarrini A. Gut microbiota in autism and mood disorders. World J Gastroenterol. 2016;22:361-8.

9. Liu YW, Liu WH, Wu CC, Juan YC, Wu YC, Tsai HP, Wang S, Tsai YC. Psychotropic effects of Lactobacillus plantarum PS128 in early life-stressed and naïve adult mice. Brain Res. 2016;1631:1-12.

10. Luo J, Wang T, Liang S, Hu X, Li W, Jin F. Ingestion of Lactobacillus strain reduces anxiety and improves cognitive function in the hyperammonemia rat. Sci China Life Sci. 2014;57(3):327-35.

11. Ait-Belgnaoui A, Durand H, Cartier C, Chaumaz G, Eutamene H, Ferrier L, et al. Prevention of gut leakiness by a probiotic treatment leads to attenuated HPA response to an acute psychological stress in rats. Psychoneuroendocrinology. 2012;37(11):1885-95.

12. Bercik P, Park AJ, Sinclair D, Khoshdel A, Lu J, Huang X, et al. The anxiolytic effect of Bifidobacterium longum NCC3001 involves vagal pathways for gut-brain communication. Neurogastroenterol Motil. 2011;23(12):1132-9.

13. Bercik P, Verdu EF, Foster JA, Macri J, Potter M, Huang X, et al. Chronic gastrointestinal inflammation induces anxiety-like behavior and alters central nervous system biochemistry in mice. Gastroenterology. 2010;139(6):2102-2112.e1.

14. Akkasheh G, Kashani-Poor Z, Tajabadi-Ebrahimi M, Jafari P, Akbari H, Taghizadeh $\mathrm{M}$, et al. Clinical and metabolic response to probiotic administration in patients with major depressive disorder: a randomized, double-blind, placebo-controlled trial. Nutrition. 2016;32(3):315-20. 
15. Messaoudi M, Lalonde R, Violle N, Javelot H, Desor D, Nejdi A, et al. Assessment of psychotropic-like properties of a probiotic formulation (Lactobacillus helveticus R0052 and Bifidobacterium longum R0175) in rats and human subjects. Br J Nutr. 2011;105(5):755-64.

16. Benton D, Williams C, Brown A. Impact of consuming a milk drink containing a probiotic on mood and cognition. Eur J Clin Nutr. 2007:61(3):355-61.

17. Cryan JF, Dinan TG. Mind-altering microorganisms: the impact of the gut microbiota on brain and behaviour. Nat Rev Neurosci. 2012;13:701-12.

18. Nagpal R, Kumar M, Yadav AK, Hemalatha R, Yadav H, Marotta F, Yamashiro Y. Gut microbiota in health and disease: an overview focused on metabolic inflammation. Benef Microbes. 2016;7:181-94.

19. Kim KA, Gu W, Lee IA, Joh EH, Kim DH. High fat diet-induced gut microbiota exacerbates inflammation and obesity in mice via the TLR4 signaling pathway. PLoS ONE. 2012;7:e47713.

20. De Lorenzo A, Soldati L, Sarlo F, Calvani M, Di Lorenzo N, Di Renzo L. New obesity classification criteria as a tool for bariatric surgery indication. World J Gastroenterol. 2016:22:681-703.

21. Oliveros E, Somers VK, Sochor O, Goel K, Lopez-Jimenez F. The concept of normal weight obesity. Prog Cardiovasc Dis. 2014;56:426-33.

22. Seo MH, Rhee EJ. Metabolic and cardiovascular implications of a metabolically healthy obesity phenotype. Endocrinol Metab. 2014;29:427-34

23. O'Connell J, Lynch L, Cawood TJ, Kwasnik A, Nolan N, Geoghegan J, McCormick A, O'Farrelly C, O'Shea D. The relationship of omental and subcutaneous adipocyte size to metabolic disease in severe obesity. PLoS ONE. 2010;5:e9997.

24. De Lorenzo A, Martinoli R, Vaia F, Di Renzo L. Normal weight obese (NWO) women: an evaluation of a candidate new syndrome. Nutr Metab Cardiovasc Dis. 2006;16:513-23.

25. Di Renzo L, Sarlo F, Petramala L, lacopino L, Monteleone G, Colica C, De Lorenzo A. Association between -308 G/A TNF-a polymorphism and appendicular skeletal muscle mass index as a marker of sarcopenia in normal weight obese syndrome. Dis Markers. 2013;35:615-23.

26. Di Renzo L, Gratteri S, Sarlo F, Cabibbo A, Colica C, De Lorenzo A. Individually tailored screening of susceptibility to sarcopenia using p53 codon 72 polymorphism, phenotypes, and conventional risk factors. Dis Markers. 2014;2014:743634.

27. De Lorenzo A, Bianchi A, Maroni P, lannarelli A, Di Daniele N, lacopino L, Di Renzo L. Adiposity rather than BMI determines metabolic risk. Int J Cardiol. 2013;166:111-7.

28. Di Renzo L, Tyndall E, Gualtieri P, Carboni C, Valente R, Ciani AS, Tonini MG, De Lorenzo A. Association of body composition and eating behavior in the normal weight obese syndrome. Eat Weight Disord. 2016;21:99-106.

29. Kootte RS, Vrieze A, Holleman F, Dallinga-Thie GM, Zoetendal EG, de Vos WM, Groen AK, Hoekstra JB, Stroes ES, Nieuwdorp M. The therapeutic potential of manipulating gut microbiota in obesity and type 2 diabetes mellitus. Diabetes Obes Metab. 2012;14(2):112-20. doi:10.1111/j.14631326.2011.01483.x (Epub 2011 Nov 22).

30. Ohland CL, Kish L, Bell H, Thiesen A, Hotte N, Pankiv E, Madsen KL. Effects of Lactobacillus helveticus on murine behavior are dependent on diet and genotype and correlate with alterations in the gut microbiome. Psychoneuroendocrinology. 2013;38(9):1738-47. doi:10.1016/j. psyneuen.2013.02.008.

31. Derogatis LR, Lipman RS, Covi L. SCL-90: an outpatient psychiatric rating scale_-preliminary report. Psychopharmacol Bull. 1973;9:13-28.

32. Cuzzolaro M, Vetrone G, Marano G, Garfinkel PE. The body uneasiness test (BUT): development and validation of a new body image assessment scale. Eat Weight Disord. 2006;11:1-13.

33. Garner DM. Eating disorder inventory-2 professional manual. 1st ed. Odessa: Psychological Assessment Resources; 1991.

34. Lohman TG, Roche AF, Martorell R. Anthropometric standardization reference manual. Champaign: Human Kinetics Press; 1998.

35. Di Renzo L, Carbonelli MG, Bianchi A, Domino E, Migliore MR, Rillo G, lacopino L, Di Daniele N, De Lorenzo A. Impact of the -174 G > C IL-6 polymorphism on bioelectrical parameters in obese subjects after laparoscopic adjustable gastric banding. J Obes. 2012;2012:208953.
36. Rizzardi M, Trombini E, Trombini G. EDI-2 eating disorder inventory-2: manuale. 1st ed. Florence: O.S. Organizzazioni Speciali; 1995.

37. Sciarretta G, Furno A, Mazzoni M, Garagnani B, Malaguti P. Lactulose hydrogen breath test in orocecal transit assessment. Critical evaluation by means of scintigraphic method. Dig Dis Sci. 1994;39:1505-10.

38. Mayer EA. Gut feelings: the emerging biology of gut-brain communication. Nat Rev Neurosci. 2011;12:453-66.

39. Dinan TG, Cryan JF. The impact of gut microbiota on brain and behaviour: implications for psychiatry. Curr Opin Clin Nutr Metab Care. 2015;18:552-8

40. Fond G, Boukouaci W, Chevalier G, Regnault A, Eberl G, Hamdani N, Dickerson F, Macgregor A, Boyer L, Dargel A, Oliveira J, Tamouza R, Leboyer M. The, "psychomicrobiotic": targeting microbiota in major psychiatric disorders: a systematic review. Pathol Biol. 2015;63:35-42.

41. Waldram A, Holmes E, Wang Y, Rantalainen M, Wilson ID, Tuohy KM, McCartney AL, Gibson GR, Nicholson JK. Top-down systems biology modeling of host metabotype-microbiome associations in obese rodents. J Proteome Res. 2009:8:2361-75.

42. Cani PD, Amar J, Iglesias MA, Poggi M, Knauf C, Bastelica D, Neyrinck AM, Fava F, Tuohy KM, Chabo C, Waget A, Delmée E, Cousin B, Sulpice T, Chamontin B, Ferrières J, Tanti JF, Gibson GR, Casteilla L, Delzenne NM, Alessi MC, Burcelin R. Metabolic endotoxemia initiates obesity and insulin resistance. Diabetes. 2007;56:1761-72.

43. Angelakis $E$, Armougom F, Million M, Raoult D. The relationship between gut microbiota and weight gain in humans. Future Microbiol. 2012;7:91-109.

44. Kato-Kataoka A, Nishida K, Takada M, Kawai M, Kikuchi-Hayakawa H, Suda K, Ishikawa H, Gondo Y, Shimizu K, Matsuki T, Kushiro A, Hoshi R, Watanabe O, Igarashi T, Miyazaki K, Kuwano Y, Rokutan K. Fermented milk containing Lactobacillus casei strain Shirota preserves the diversity of the gut microbiota and relieves abdominal dysfunction in healthy medical students exposed to academic stress. Appl Environ Microbiol. 2016:82:3649-58.

45. Drissi F, Raoult D, Merhej V. Metabolic role of lactobacilli in weight modification in humans and animals. Microb Pathog. 2016. doi:10.1016/j. micpath.2016.03.006

46. De Lorenzo A, Del Gobbo V, Premrov MG, Bigioni M, Galvano F, Di Renzo L. Normal-weight obese syndrome: early inflammation? Am J Clin Nutr. 2007:85:40-5.

47. Dinan TG, Stilling RM, Stanton C, Cryan JF. Collective unconscious: how gut microbes shape human behavior. J Psychiatr Res. 2015;63:1-9.

48. Dinan TG, Cryan JF. Melancholic microbes: a link between gut microbiota and depression? Neurogastroenterol Motil. 2013;25:713-9.

49. Schmidt K, Cowen PJ, Harmer CJ, Tzortzis G, Errington S, Burnet PW. Prebiotic intake reduces the waking cortisol response and alters emotional bias in healthy volunteers. Psychopharmacology. 2015;232:1793-801.

50. Bailey MT, Dowd SE, Parry NM, Galley JD, Schauer DB, Lyte M. Stressor exposure disrupts commensal microbial populations in the intestines and leads to increased colonization by Citrobacter rodentium. Infect Immun. 2010;78:1509-19.

51. Bailey MT, Dowd SE, Galley JD, Hufnagle AR, Allen RG, Lyte M. Exposure to a social stressor alters the structure of the intestinal microbiota: implications for stressor-induced immunomodulation. Brain Behav Immun. 2011;25:397-407

52. Agostini S, Goubern M, Tondereau V, Salvador-Cartier C, Bezirard V, Lévèque $M$, Keränen $H$, Theodorou V, Bourdu-Naturel S, Goupil-Feuillerat $\mathrm{N}$, Legrain-Raspaud S, Eutamene $\mathrm{H}$. A marketed fermented dairy product containing Bifidobacterium lactis CNCM I-2494 suppresses gut hypersensitivity and colonic barrier disruption induced by acute stress in rats. Neurogastroenterol Motil. 2012;24:376.

53. Kamiya T, Wang L, Forsythe P, Goettsche G, Mao Y, Wang Y, Tougas $G$, Bienenstock J. Inhibitory effects of Lactobacillus reuteri on visceral pain induced by colorectal distension in Sprague-Dawley rats. Gut. 2006;55:191-6. 